\title{
然
}

\author{
Álvaro Angulo Maenza*
}

\section{UNA NUEVA ERA PARA LA INDUSTRIA EUROPEA}

El sector industrial se ha mostrado siempre como uno de los grandes motores de crecimiento de las economías. De hecho, gracias a la Revolución Industrial, Europa vivió un cambio social y económico sin precedentes. En la actualidad, esta importancia no ha disminuido y, conscientes de ello, China y Estados Unidos se han convertido en los dos grandes bloques industriales del momento, con una Europa que, al terciarizarse, dejó que su industria perdiera importancia a principios de este siglo. Ahora, en un entorno con constantes cambios y con China y Estados Unidos como las grandes potencias con las que competir, la reacción europea debe ser ágil, impulsando definitivamente las políticas necesarias para que el sector recobre su fuerza, revirtiendo la situación y aprovechando las amplísimas oportunidades que se presentan en un futuro que ya ha llegado.

Palabras clave: cadenas de valor estratégicas, baterías, industria 4.0, inteligencia artificial, campeones nacionales.

Clasificación JEL: L0, L1, L52.

\section{Un poco de historia}

Un fuerte crecimiento de la economía de un país pasa necesariamente por un sector exterior abierto, puesto que los beneficios del comercio internacional se han mostrado mucho mayores que los costes que pudieran provocar. Históricamente, se ha constatado cómo un país autárquico, cerrado al exterior (nosotros fuimos testigos tras el final de la Guerra Civil) ha generado grandes retrasos económicos y, por ende, sociales.

\footnotetext{
* Funcionario del Cuerpo de Diplomados Comerciales del Estado. Ministerio de Economía y Empresa.

Versión de septiembre de 2019.

DOI: https:/doi.org/10.32796/bice.2019.3117.6925
}

Un sector industrial sólido y avanzado juega un papel relevante para que un país en un entorno globalizado como el actual se pueda beneficiar del comercio exterior; y es que la industria es uno de los motores de la economía mundial, tanto en productividad como en innovación. Concretamente, a la industria se le debe más del $80 \%$ de las exportaciones europeas y de la investigación e innovación privadas, emplea prácticamente al $24 \%$ de los trabajadores europeos y crea entre 0,5 y 2 trabajos en otros sectores por cada puesto de trabajo industrial adicional.

Esta importancia de la industria se originó con la Revolución Industrial (siglos XVIII y XIX), la cual supuso el cambio de paradigma más importante desde el Neolítico, cuando la aparición $\triangleright$ 
de la agricultura, la capacidad para domesticar animales y el dominio del fuego propiciaron una sociedad sedentaria que empezó a organizarse en pequeños asentamientos. Por su parte, la Revolución Industrial provocó, entre otros, el éxodo del campo a las ciudades, una gran explosión demográfica, un aumento de la esperanza de vida, del poder adquisitivo, de la productividad laboral y de la producción, en el que el transporte de las mercancías a una mayor velocidad (especialmente el de las más pesadas) se facilitó gracias al ferrocarril, permitiendo un mayor volumen de intercambios comerciales. En definitiva, se asentó el capitalismo y la economía de mercado. La sociedad cambió.

Con el paso del tiempo, las economías modernas se han ido terciarizando en detrimento de los sectores primario y secundario, especialmente a partir del año 20001. Esta terciarización tiene elementos positivos, puesto que el desarrollo de actividades tales como servicios de consultoría, diseño, marketing y transporte contribuyen al crecimiento de actividades innovadoras que cada vez son más utilizadas en la industria e incrementan la productividad del sector. Sin embargo, tras la larga recesión económica iniciada en 2008, se ha subrayado el carácter estratégico que tiene el sector industrial, lo cual queda reflejado en el enfoque que la Unión Europea ha ido adoptando a partir de ese momento como apoyo a las políticas industriales de los Estados miembros. Una de estas primeras aproximaciones hacia esta nueva orientación vino de la mano del Tratado de Funcionamiento de la Unión Europea, en el que se establece, entre otros, que la Unión se compromete a llevar a cabo acciones de apoyo a las políticas industriales de sus Estados miembros

El valor añadido de la industria en el PIB era del $21 \%$ en 1995 y del $18 \%$ en 2005. y a reforzar la competitividad de la industria de la Unión, todo ello debido a que, a pesar de que el tejido industrial de la UE está consolidado, debemos mantener nuestro liderazgo en la materia, especialmente ante los retos planteados a corto plazo. Estos nuevos elementos que entran en juego se caracterizan por la rapidez con que se producen los cambios tecnológicos (especialmente en robótica, internet de las cosas, inteligencia artificial y sistemas energéticos y medioambientales), que, a su vez, propician modelos empresariales inexistentes en un pasado cercano, difuminando poco a poco la diferencia entre el sector industrial y los servicios.

Por ello, ya en 2014, la Unión Europea abogó por relanzar la industria bajo la comunicación de la Comisión titulada Por un renacimiento industrial europeo, cuyo objetivo es que el sector represente el $20 \%$ del PIB en 2020. Actualmente, la media de la UE se sitúa en un $17,5 \%$, pero el verdadero problema es que, de los países más fuertes, solo Alemania $(23,1 \%)$ está por encima de ese valor. Italia se sitúa justo en la media y España (16,3\%), Portugal $(15,9 \%)$, Dinamarca $(15,6 \%)$ y, sobre todo, Países Bajos (13,3\%), Grecia (13,1\%) Reino Unido (12,6\%) y Francia (12,2\%) se sitúan por debajo. En este contexto, en el que el objetivo de alcanzar el $20 \%$ del PIB en 2020 se muestra un tanto utópico, Alemania ha propuesto (a través de su ministro de Economía, Peter Altamier) seguir avanzando hacia la consecución de esta meta, incrementando su participación del PIB industrial hasta el $25 \%$ y el $20 \%$ en el conjunto de la UE en $2030^{2}$.

Esta tendencia se confirma en el ámbito internacional, con una cada vez mayor importancia del sector industrial: China, con un peso $D$

2 National Industrial Strategy 2030: Strategic guidelines for a German and European industrial policy. 


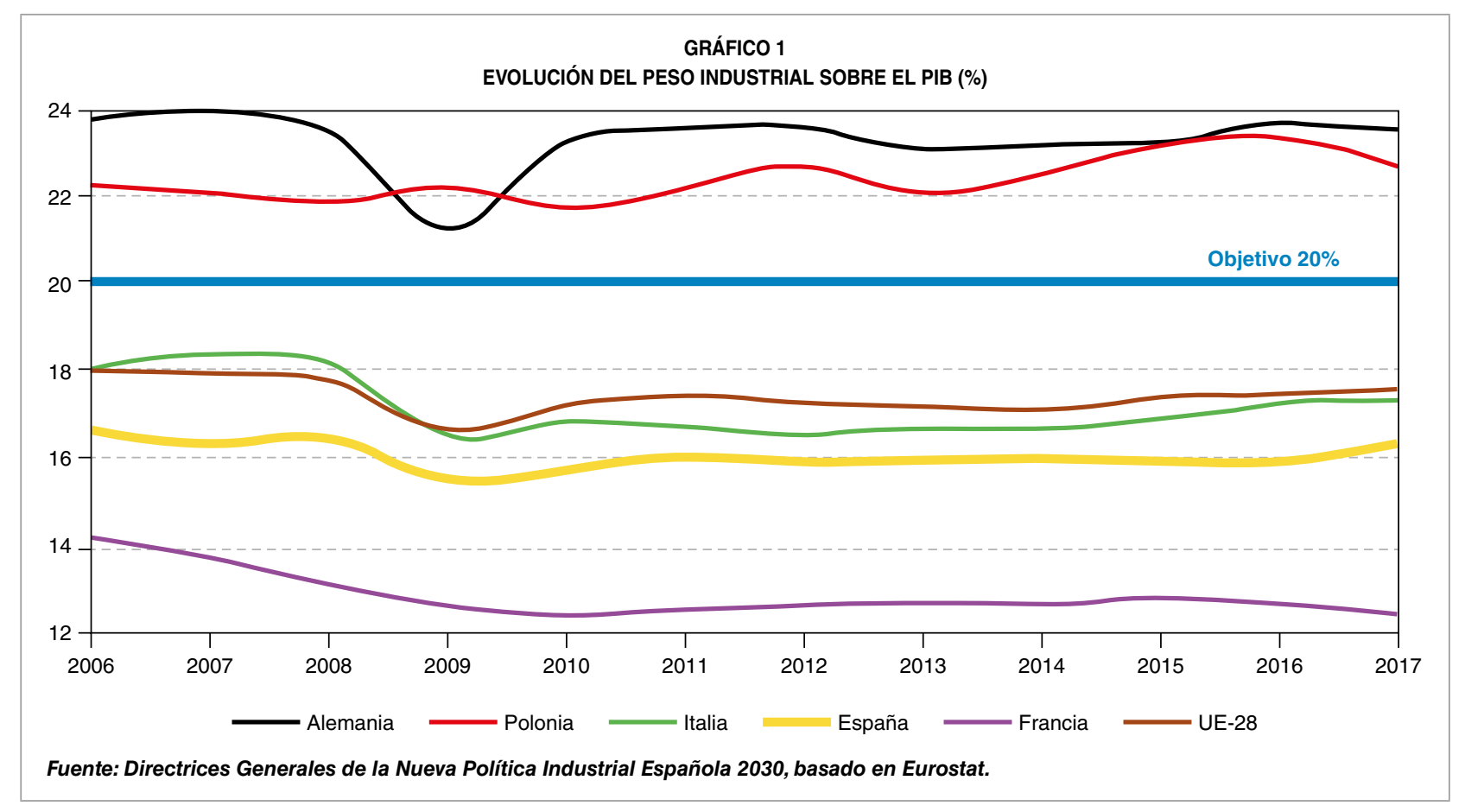

de más del $40 \%$ del PIB, Corea del Sur (37,3\%), Rusia (33,3\%), Chile (30\%), Japón (29,3\%) y Estados Unidos (19,1\%) son ejemplos de ello.

Ante este contexto, la Unión Europea debe adaptarse e innovar permanentemente, mientras el conjunto del tejido industrial debe asumir los cambios introducidos en los últimos tiempos, con un especial énfasis en la digitalización y la economía circular, que se configuran como las señas identitarias de la industria europea frente a sus competidores. Estas exigencias fueron ya identificadas en la Comunicación de 2017 de la Comisión: Invertir en una industria inteligente, innovadora y sostenible. Estrategia renovada de política industrial de la $U E$, en la que se subraya la necesidad de adaptar y reforzar la industria europea a la nueva era industrial a través de inversiones y apoyos para un mercado único más justo y profundo, modernizando la industria de cara a la era digital y fortaleciendo la asociación entre los Estados miembros, las regiones, las ciudades y el sector privado.

\section{Una nueva era}

En vista de lo anterior, no cabe duda de que la estructura económica está en constante evolución: por una parte, porque los mercados mundiales están inmersos en un veloz proceso de transformación con una aceleración de la globalización en términos generales, y mayores y más rápidas innovaciones tecnológicas; por otra, por la aparición de medidas proteccionistas, derivadas más bien de estrategias políticas y el abandono de ciertos acuerdos internacionales.

Entre estas amenazas destacan el brexit, el intervencionismo económico chino (lo que se conoce como capitalismo de Estado) y la guerra comercial de EE UU con China y, más recientemente, con Europa, imponiendo barreras arancelarias al libre comercio. Las economías cuyo comercio exterior esté especialmente enfocado en alguno de estos países pueden sufrir peores consecuencias; sin embargo, para España, si bien la bajada de tipos por parte $\triangle$ 
de la Fed puede afectar al conjunto de nuestras exportaciones por la apreciación del euro frente al dólar, la principal preocupación viene dada por la importancia de la economía China y los efectos de una posible salida del Reino Unido de la Unión Europea. Si finalmente esto ocurre y no viene acompañado de un acuerdo comercial amistoso entre ambos, las exportaciones españolas y la inversión directa recibida podrían disminuir debido a la depreciación de la libra. Además, se produciría un incremento de los costes para toda la industria a causa de la imposición de medidas arancelarias, así como de las posibles consecuencias que tenga la restricción de la cláusula de reconocimiento mutuo entre los países de la Unión.

No obstante, los retos a los que se enfrenta la industria no solo derivan de este posible debilitamiento de la integración regional, puesto que de la competencia más «tradicional» emanan los principales retos para la industria española:

- Nuestra ventaja competitiva basada en bajos salarios y reducidos costes productivos forma parte del pasado, dejando de ser una de nuestras fortalezas debido al desarrollo del sector industrial en otros Estados miembros de adhesión más reciente (República Checa, Polonia o Rumanía) y en países emergentes. Consecuencia de ello, España ha ido desarrollando paulatinamente un nuevo modelo productivo centrado en la utilización de nuevas tecnologías y calidad, acumulado conocimiento y una red de proveedores que le ha permitido mantener su desarrollo industrial. No obstante, incluso esta nueva caracterización puede suponer un riesgo, puesto que países como China han logrado incrementar su experiencia sobre la adaptación y el desarrollo de nuevas tecnologías (como es el caso de los progresos realizados en la tecnología del vehículo eléctrico), a través de sus propios desarrollos o debido a joint ventures o adquisiciones de empresas europeas punteras. Esta nueva tendencia es solo compensada en parte por los incrementos en los salarios y gastos sociales en los países emergentes.

- En conexión con el punto anterior, se ha constatado que, en los últimos años, Europa, a pesar de adoptar procesos productivos más intensivos en tecnología, no ha sido capaz de liderar el desarrollo de las mismas. Las tecnologías de la comunicación, la robótica, los smartphones, las tablets, las baterías o los materiales de fibra de carbono son principalmente producidos y ensamblados en países asiáticos. Pero, además, esta situación se verá acrecentada por el acceso y la explotación de las materias primas utilizadas en estos desarrollos, las cuales serán uno de los factores críticos en la competitividad de las empresas. A pesar de lo anterior, esta escasez de materiales puede acabar siendo una oportunidad, ya que pueden incentivar el desarrollo de productos más funcionales, con menor peso, menor impacto medioambiental y de mayor eficiencia energética.

- Las compañías globales de tecnología e internet se están desarrollando casi en exclusiva en EE UU, China, Japón y Corea del Sur. Incluso algunas marcas que el público general identifica como europeas en realidad desarrollan sus productos en países asiáticos o están financiadas por capital extranjero. En este $\triangleright$ 
área hay un claro margen de actuación, sobre todo de la mano del desarrollo de la inteligencia artificial que tiene un amplio abanico de aplicaciones prácticas, por lo que España deberían dedicar gran parte de sus esfuerzos en este campo.

- Los países más avanzados y desarrollados tienen un importante sector industrial. Sin embargo, a medida que las economías siguen madurando, los servicios continúan creciendo con un incremento en su productividad y especialización en actividades de alta cualificación que antes no existían en el sector. Esto lleva a una terciarización de la industria y a un descenso en el empleo puramente industrial, generando un trade-off industria-servicios. Así, el mayor uso de la tecnología y la mayor automatización de los procesos de producción harán que estos sean menos intensivos en mano de obra, pero aumentará el requerimiento de la altamente cualificada. Esta mano de obra cualificada permite, además, adaptar la producción a las nuevas tendencias de consumo con una cada vez mayor importancia de los productos hechos a medida.

- Esta adaptación del producto determinará, igualmente, el modo en que la industria opera. Las diferencias entre los distintos mercados a nivel global y una creciente clase media en los países emergentes supondrán grandes retos para el sector, que tendrá que producir distintos productos para distintos mercados, reaccionar a los cambiantes gustos de los clientes, adaptar distintas políticas de precios, diseñar medidas variadas de atracción de clientes..., que no harán sino complicar el proceso productivo.
- Precisamente todo lo anterior tiene sentido en el contexto europeo en general y en el español en especial. El tejido empresarial está fundamentalmente compuesto por pymes, y esto tiene un claro impacto sobre la inversión en $1+D+i$, la propensión exportadora, etcétera. La gran importancia para nuestro tejido industrial ha quedado patente con la aprobación, el pasado mes de abril, del Marco Estratégico en Política de Pequeña y Mediana Empresa 2030, el cual recoge líneas de actuación horizontales con un gran énfasis en la sostenibilidad, digitalización, innovación, internacionalización, crecimiento y flexibilización de los instrumentos de financiación a los que tienen acceso. A escala europea, también se tiene conciencia sobre estos retos, por lo que se está promoviendo la agrupación de empresas, así como el apoyo a la creación de cadenas de valor estratégicas en las que integrar empresas a lo largo de todo el ciclo del producto, desde la obtención de la materia prima para su fabricación, pasando por todo su proceso productivo, hasta su reutilización o reciclaje, con especial énfasis en aquellas tendencias o retos a los que se enfrentarán las industrias europeas de aquí a 2030.

En la actualidad, la Comisión Europea ha identificado seis cadenas de valor estratégicas ${ }^{3}$ :

1. Vehículos conectados, automatizados y eléctricos.

\section{Salud inteligente.}

3 La Comisión presentó el 6 de febrero de 2019, en las Jornadas de la Industria de la UE, las recomendaciones preliminares del grupo de expertos sobre cadenas de valor estratégicas. 
3. Industrias hipocarbónicas.

4. Tecnologías y sistemas de hidrógeno.

5. Internet industrial de las cosas.

6. Ciberseguridad.

Todas ellas complementarán las iniciativas ya en curso para reforzar las cadenas de valor de baterías, microelectrónica e informática de alto rendimiento.

La función de estas cadenas de valor es la de detectar empresas que estén trabajando en un ámbito en concreto de los expuestos anteriormente e involucrarlos en los proyectos declarados de interés comunitario que puedan encajar dentro de una determinada alianza. Por su parte, las cadenas de valor estratégicas permitirán que los interesados en el proyecto presenten sus ideas para su clasificación como Proyectos Importantes de Interés Común Europeo (PIICE), permitiéndoles beneficiarse de ayudas de Estado admitidas por los reglamentos comunitarios. Una de las cadenas de valor en las que más énfasis se está poniendo es la del desarrollo de baterías, por su potencial para crear puestos de trabajo de alto valor añadido y aumentar la producción. Pueden convertirse en un importante elemento impulsor de la competitividad y liderazgo de la Unión Europea, en particular en el sector del automóvil, de gran importancia para Europa y España.

\subsection{Retos para la industria automovilística}

Como decíamos, la industria del automóvil es clave en Europa y España: nuestro país ocupa el noveno puesto del ranking mundial como productor de automóviles y es segundo en Europa, por detrás de Alemania. En conjunto, la industria española de la automoción, incluyendo a los fabricantes de automóviles y de componentes (además de los relacionados del sector, como los seguros o la distribución), representa aproximadamente el $10 \%$ del PIB, da trabajo a dos millones de personas (más del $9 \%$ de la población activa) y supone el $19 \% \triangleright$

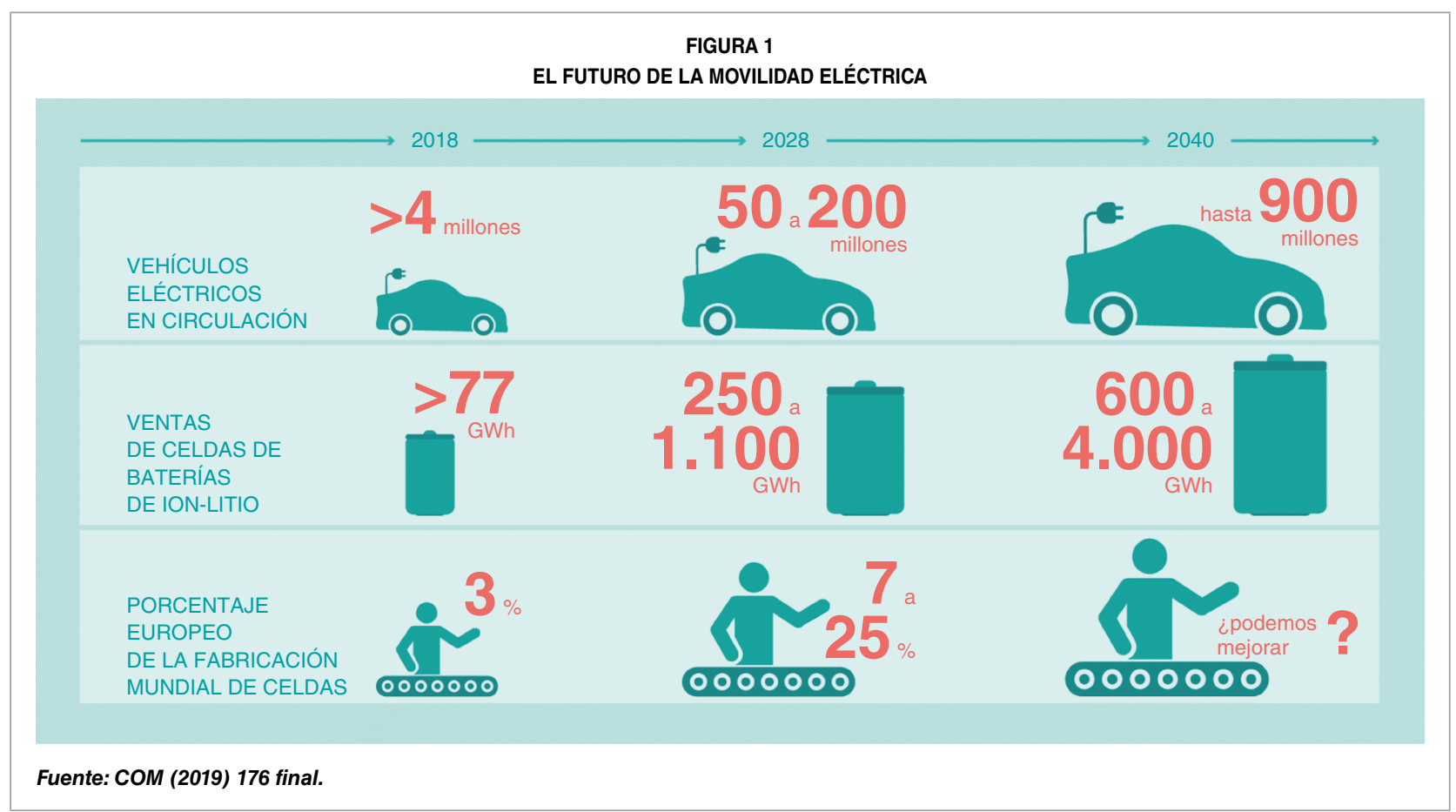


de las exportaciones del país ${ }^{4}$, generando un efecto multiplicador sobre el resto de ramas de la actividad económica. Sin embargo, el conjunto de la industria se enfrenta a verdaderos retos, no solo futuros, sino actuales: desde el desarrollo de vehículos de nulas o bajas emisiones (vehículo eléctrico o de hidrógeno), pasando por nuevas modalidades de movilidad alternativas (carsharing, carpooling, uso de patinetes), hasta las innovaciones en conducción autónoma.

Precisamente, la preocupación por el desarrollo de las baterías ha empujado a la Unión Europea al diseño de un plan de acción estratégico y al desarrollo de la cadena de valor estratégica de vehículos conectados, automatizados y eléctricos. Según las previsiones, el valor potencial del mercado europeo puede alcanzar los 250.000 millones de euros anuales a partir de 2025. Otros datos que respaldan esta tendencia son las proyecciones en la demanda mundial de baterías que aumentará desde los 78 GWh actuales hasta los $660 \mathrm{GWh}$ en 2023, $1.100 \mathrm{GWh}$ en 2028 y hasta 4.000 GWh en 2040; se espera que se pase de los algo más de 4 millones de vehículos eléctricos que hay actualmente en circulación a nivel mundial, a los entre 50-200 millones en 2028 y hasta los 900 millones en 2040 (Comisión Europea, 2019); y, por último, se calcula que en Europa se deberán construir de veinte a treinta gigafábricas ${ }^{5}$ para la producción de celdas exclusivamente, por lo que deberá

\footnotetext{
4 Datos recogidos del Informe Anual de ANFAC (2018): Adelantando el futuro.

5 El término gigafábrica ha comenzado a utilizarse tras la construcción, por parte de Tesla, de una planta de baterías (la construcción se inició en 2014 y estará lista en 2020, aunque ya se utilizan las secciones terminadas) para cubrir la demanda de las mismas en base a las previsiones. El término se usa para denominar fábricas de gran superficie (con gran aprovechamiento de economías de escala al concentrar la producción) y que, además, son respetuosas con el medio ambiente al estar alimentadas por energías renovables. Tesla posee otra gigafábrica en el estado de Nueva York, una tercera en Shanghai (que comienza a operar a finales de año) y se va a construir una cuarta en Europa con apertura prevista a finales de 2021 (probablemente en Alemania).
}

reforzarse todo el ecosistema que orbita a su alrededor.

Así, si tomamos conciencia de que el coche eléctrico es el futuro de la movilidad, España no solo debe atraer la producción de este tipo de vehículos con el objeto de conservar el peso actual del sector, sino tratar de que los centros productivos necesarios para la producción de baterías se instalen en nuestro país, ya que corremos el riesgo de convertirnos en meros ensambladores y de que los nuevos trabajos que se creen, debido a las innovaciones tecnológicas que están por venir y el consiguiente aumento de la productividad, no recalen necesariamente en aquellos países o regiones donde los trabajos tradicionales desaparezcan (el nuestro). Por tanto, si tenemos en cuenta que gran parte de nuestra producción de vehículos se basa en tecnologías de combustión internas (diésel y gasolina suman más del $90 \%)^{6}$, hay un riesgo patente de que España pierda una parte importante de valor añadido en la industria si no es capaz de posicionarse ante los nuevos retos tecnológicos del sector. Además, se estima que el paso a una movilidad eléctrica podría generar en nuestro país una pérdida de 40.000 empleos y reducir el PIB en un 1,2\% (Scholtes, 2019) ${ }^{7}$. De hecho, estos efectos ya han empezado a notarse: hasta el mes de junio la producción de vehículos acumula un descenso del $5,6 \%$ respecto al mismo periodo de 2018 , sumando ocho meses de caídas. Por ello, es fundamental atraer nuevas inversiones y ampliar las exportaciones a países como China o India, que siguen siendo mercados con gran potencial a pesar del incremento exponencial de la demanda en los últimos años ${ }^{8}$.

\footnotetext{
6 Datos procedentes de las notas de producción y exportación elaboradas por ANFAC.

7 Impacto del vehículo eléctrico en la industria española: disrupción económica en ciernes.

8 Datos procedentes de las notas de producción y exportación elaboradas por ANFAC.
} 
Otro aspecto de gran importancia en la producción de las baterías es la procedencia de las materias primas; y es que una gran parte de los materiales usados en estas tecnologías (tierras raras), como el litio, el cobalto y el grafito, pertenecen mayoritariamente a Australia, Congo y China, respectivamente. A su vez, este último es el principal suministrador de materiales transformados utilizados para la fabricación de baterías: cátodo, ánodo y electrolito.

Queda claro que la Unión Europea debe diversificar en la medida de lo posible sus proveedores de materias primas para no depender de un único país proveedor, garantizando así un suministro sostenible y seguro, y disminuyendo paulatinamente esa dependencia mediante la recuperación y el reciclado de los materiales más valiosos de las baterías. Este aspecto es muy importante, puesto que la provisión de un material por parte de un solo país puede tener consecuencias graves para la economía. Ejemplo de ello es la reciente amenaza china de cortar el suministro de tierras raras, en respuesta a los aranceles impuestos por EE UU de los productos procedentes del país asiático. Aquí es donde entra en juego la importancia de la economía circular, que puede diseñarse de tal modo que minimice riesgos, incremente las oportunidades laborales y suponga una ventaja competitiva al ahorrar costes.

Igualmente importante en el desarrollo de las baterías es el planteamiento sobre qué tipo de materiales se deberían emplear. Dado que el desarrollo de las baterías de litio en estado líquido (iones de litio) ha venido de la mano de China y Corea del Sur, una estrategia factible podría configurarse en el desarrollo de nuevas generaciones de baterías basadas en posibilidades tales como el litio en estado sólido, zinc-aire, magnesio..., que dependerán, a su vez, de los materiales que se encuentren en nuestro suelo. En este sentido cabe destacar que España (concretamente Extremadura) posee, según los responsables del consorcio que pretende explotar la mina de litio y producir posteriormente hidróxido de litio (Infinity Lithium), el segundo enclave con mayores posibilidades en Europa, lo que podría traducirse según sus datos en unos ingresos de 5.500 millones de euros durante los treinta años de explotación previstos, la creación de mil puestos de trabajo directos e indirectos al año y dar cabida a la producción de baterías para diez millones de vehículos en total.

Por último, y no por ello menos importante, se deben tener en cuenta las nuevas modalidades de movilidad que se están expandiendo en los países desarrollados, principalmente en las grandes ciudades, dadas la congestión del tráfico y la elevada contaminación. Todo lo anterior, junto con las mejoras en los sistemas de transporte público, han propiciado el desarrollo de modelos tales como el carsharing, que permite la sustitución de vehículos particulares (generalmente más obsoletos y, por tanto, contaminantes) por otros de uso compartido de cero emisiones, que están en circulación la gran parte del tiempo. Precisamente todos estos problemas, junto a la incertidumbre sobre el futuro de las tecnologías de combustión «tradicionales", pronostican un menor uso del vehículo en propiedad para convertirse en un servicio bajo demanda.

Pese a todas las amenazas identificadas con anterioridad, el diseño de una buena hoja de ruta para los próximos años aprovechando las oportunidades potenciales, un buen posicionamiento de nuestro país para el desarrollo de la nueva generación de baterías y la continuidad de incentivos a la oferta y la demanda de vehículos de energías alternativas en nuestro territorio podrían suponer un verdadero $\triangleright$ 
revulsivo para la industria automovilística española, asegurando así su futuro.

\subsection{Industria 4.0: ¿queremos campeones o un tejido equilibrado?}

Otro de los aspectos fundamentales para la industria es su digitalización. De hecho, una de las cadenas de valor estratégicas identificadas por la Unión Europa, y que hemos citado previamente, es el internet industrial de las cosas y la ciberseguridad. Esta identificación venía precedida por la Comunicación de la Comisión de septiembre de 2017 titulada Invertir en una industria inteligente, innovadora y sostenible. Estrategia renovada de política industrial de la UE.

Tal y como hemos apuntado, el futuro de la industria pasa por aplicar las revoluciones de internet a los procesos productivos, en especial aquellos relacionados con la inteligencia artificial y el internet de las cosas, puesto que permiten incrementar la productividad y la eficiencia. El problema radica en que si no se aplican estos cambios tecnológicos, no solo se deja de ganar productividad, sino que en términos comparativos se pierde $\mathrm{y}$, por tanto, disminuye la cuota de mercado, poniendo en peligro la rentabilidad de la empresa que no haya adoptado estas innovaciones.

La asunción de este tipo de avances es más complicada y paulatina respecto a las actividades más tradicionales de nuestra industria al ser necesario asumir importantes costes: no solo aquellos necesarios para la implantación de la innovación en sí, sino también los relativos a la formación del personal para la correcta utilización de dichos avances. Adicionalmente, esta cuestión no solo afecta a las pymes, sino, lo que es más importante, al conjunto de empresas europeas, especialmente a aquellas que son muy valiosas en nuestra economía pero que tienen un tamaño relativamente modesto respecto a las grandes corporaciones chinas y/o estadounidenses y que, por tanto, compiten en condiciones de inferioridad.

Ante esta problemática se abre el debate sobre la conveniencia de frenar o apoyar la aparición de campeones nacionales, por el importante potencial que representan en este campo. En defensa de esta idea, se ha constatado que incluso para poder ofrecer ciertos productos o servicios (como puede ser, por ejemplo, la fabricación de aeronaves) se necesitan grandes cantidades de capital; necesidad que se ve incrementada para que la empresa participe en los mercados mundiales. En España y en Europa ya existen estos campeones nacionales, pero se identifican en sectores relativamente tradicionales que, si bien también tienen una gran importancia en los procesos de $\mathrm{I}+\mathrm{D}+\mathrm{i}$, no suponen tanto en comparación con las industrias tecnológicas puras ${ }^{9}$. Ejemplos de ello son Banco Santander, Inditex, Gestamp, Airbus, Siemens, Total...

Sin embargo, y de acuerdo con datos de Bloomberg, de entre las veinte compañías mundiales con más capitalización en bolsa solo hay una que pertenece a la Unión Europea: Royal Dutch Shell, empresa holandesa dedicada a los hidrocarburos y refinado de gasolinas. Por lo tanto, si como país o como Unión no se incentiva la creación de grandes empresas, se pierde la posibilidad de competir con garantías en el mercado exterior, se dificulta el liderazgo en el desarrollo de nuevas tecnologías y se es más vulnerable frente a $D$

\footnotetext{
$9 \mathrm{Y}$ decimos «no tanto» puesto que en la industria del automóvil, aunque pueda entenderse como más tradicional, cada vez tienen mayor importancia los avances tecnológicos (cámaras $360^{\circ}$, limitadores de velocidad, faros LED adaptativos, conducción autónoma), de tal manera que los fabricantes destinan alrededor del $5 \%$ de su gasto a $I+D+i$.
} 
potenciales adquisiciones foráneas de nuestra industria nacional, especialmente si actúan en sectores estratégicos.

Especialmente acuciante es el caso de las empresas tecnológicas que - a pesar de que en la Unión Europea nos encontramos con alguna como Spotify, Shazam, Skype o Kinghan sido adquiridas o se están desarrollando en China, Corea del Sur y EE UU; sobre todo aquellas dedicadas a las áreas de telecomunicaciones (Huawei, Xiaomi, Samsung, LG, Apple) e internet (WeChat, Tencent, Alibaba, Amazon, Facebook, Google). Precisamente estas empresas tecnológicas y de internet invierten aún más recursos en la investigación y desarrollo de aplicaciones en inteligencia artificial, digitalización, conducción autónoma y biotecnología, que se configuran como los cimientos de crecimiento económico, generando mayor valor añadido, por lo que la ganancia es doble.

Sin embargo, en la Unión Europea la formación de campeones nacionales también supone ciertos condicionantes. Uno de los métodos para formar estas grandes empresas es mediante concentraciones entre empresas, las cuales deben ser compatibles con las normas de competencia, de forma que las autoridades no vean amenaza alguna para el correcto funcionamiento de los mercados domésticos. Esto plantea un amplio debate sobre las ventajas e inconvenientes que estos campeones suponen para la economía de la Unión, ya que su capacidad de liderazgo puede comprometer la supervivencia de sus competidores más pequeños, al tiempo que permitirían hacer frente a los ya citados competidores asiáticos o estadounidenses.

Como soluciones alternativas, se podría optar por algo más equilibrado y promover un aumento generalizado en el tamaño de la industria y que una gran parte de las pymes adopten la digitalización en sus procesos industriales, de ahí nuestra pregunta inicial sobre un tejido equilibrado o alguien que destaque en detrimento del resto. Parece que la Comisión se decide más por un equipo equilibrado, por lo que ha puesto en marcha la estrategia sobre la «digitalización de la industria europea», poniendo especial énfasis en una mayor digitalización de la industria. También se está invirtiendo en la implantación de polos de innovación digital y otros centros tecnológicos para ofrecer a las empresas de la Unión Europea más servicios de apoyo y mejor coordinados.

\section{Y a pesar de todo, ¿la historia se repite?}

La Revolución Industrial nació en Europa y cambió el mundo en el que vivimos. Desde entonces, el sector secundario ha sido esencial en la productividad y en la innovación de la economía, y sigue siéndolo. Actualmente, estamos a las puertas de una nueva era industrial (Tercera Revolución Industrial) ${ }^{10}$, si no ha comenzado ya, quizás sin la misma entidad que aquellas de los siglos XIx y xx pero que va cambiando nuestra manera de interactuar, trabajar, circular, vivir. El uso de internet se ha expandido a todos los campos de nuestra vida y las innovaciones no paran de llegar con una rapidez inusitada. Todo y todos estaremos interconectados. Estos cambios ponen en cuestión la forma que la industria había mantenido hasta ahora, siendo necesaria su transformación, adoptando y adaptando los nuevos desarrollos tecnológicos que van apareciendo. Como todo cambio, este tiene un origen y desarrollo que ahora $\triangleright$

10 El concepto nació en 2011 de la mano de Jeremy Rifkin, basándose en los cambios producidos en la generación de energía y en las tecnologías de la información. 
parece localizarse en EE UU y los países asiáticos, con China a la cabeza. Y es precisamente ésta la principal amenaza para la industria europea, la que otrora fuera catalizadora del sector industrial y los cambios sociales.

Así, en el contexto de la creciente influencia económica y política de China, la Unión Europea ha reaccionado paulatinamente. En 2018, presentó varias propuestas de modificación de reglamentos del Parlamento Europeo y del Consejo con la intención de regular, fundamentalmente, el acceso a productos y servicios de terceros países al mercado de la Unión en los ámbitos de contratación pública, de inversiones directas extranjeras y de importaciones que sean objeto de dumping por países no miembros, con el objetivo de proteger los intereses de la Unión, penalizando, si fuera necesario, a aquellos terceros países que registren medidas o prácticas discriminatorias.

De manera más concreta, para el desafío procedente de China, en marzo de 2019, se presentó una comunicación titulada UE-China: una perspectiva estratégica, con diez acciones concretas para afinar el enfoque de Europa en su relación con el país asiático, las cuales se resumen en tres grandes objetivos:

- Profundizar en los intereses comunes de la UE y China a nivel mundial.

- Perseguir condiciones recíprocas y equilibradas que rijan las relaciones económicas entre ambos países.

- Reforzar las políticas nacionales y la base industrial europea a fin de mantener su prosperidad y valor a largo plazo.

Adicionalmente a estas medidas más generalistas de corte liberal, la Comisión Europea apuesta por un refuerzo de la política industrial que se basaría en tres pilares: 1) $1+D+i, 2)$ mejores condiciones en el mercado y 3) mayor acceso a capital humano y habilidades. De tal manera que propone políticas de apoyo a empresas emergentes, políticas de atracción de talento, integración y cooperación, etcétera. Todo ello basado en que, para luchar de tú a tú con la economía estatalista china, se necesita un claro compromiso público para apoyar proyectos de investigación, educación, infraestructuras y tecnologías innovadoras.

Ahora bien, a partir de aquí quedan en el tintero numerosos interrogantes que afectarán de manera directa al devenir del sector industrial en el corto y largo plazo. En primer lugar cabe pensar en si esta orientación que ha tomado la Comisión en los últimos meses se mantendrá tras la configuración de la nueva estructura que está a punto de echar a andar a falta de la aprobación última de la Eurocámara. En principio, todo parece indicar que será así, ya que Von der Leyen (nueva presidenta de la Comisión Europea) ha revelado en la presentación de la nueva estructura de la Comisión que sus tres vicepresidentes ejecutivos (que forman parte del total de los ocho vicepresidentes) desempeñarán una doble función: serán comisarios a la vez que vicepresidentes encargados de una de las tres áreas fundamentales de la agenda de la presidenta electa: cambio climático, entorno digital y competencia y economía (estabilidad financiera y mercados de capitales) ${ }^{11}$.

En segundo lugar, no podemos olvidar que, en lo que a industria se refiere, la Comisión Europea dicta una serie de apoyos y recomendaciones a los Estados miembros, siendo estos los que tienen la última palabra en el diseño y aprobación de su propia política industrial. $\triangleright$

11 Con base en el comunicado de prensa de la Comisión Europea de 10 de septiembre de 2019. 
En consecuencia, la gran pregunta que se plantea aquí es si la política que se va a llevar a cabo será como bloque o por país.

Por una parte, Alemania ha propuesto fortalecer y revitalizar la economía de mercado como la mejor respuesta a los cambios industriales y tecnológicos; es decir, la intervención del Estado debería ser excepcional, yendo de la mano de las empresas tan solo en los aspectos fundamentales, conjugando los intereses de estas con los del resto de la sociedad, pero dándoles la confianza y el margen de maniobra suficientes para asegurar un futuro prometedor en el sector y en la economía, por lo que la necesidad de una intervención estatal debería estar únicamente justificada en aquellos casos donde las demás opciones se hayan mostrado inadecuadas.

Fruto de la anterior propuesta se deriva el manifiesto a favor de una política industrial europea para el siglo xxI firmado por Francia y Alemania, y que contiene cuatro grandes mensajes: 1) necesidad de una política industrial europea -lo que parece resolver la pregunta sobre si se actuará como bloque-; 2) invertir en innovación tecnológica, especialmente en inteligencia artificial; 3) reformar las leyes de competencia con el objetivo de competir a nivel mundial; y 4) desarrollar mecanismos de protección frente a la competencia desleal de fuera y reforzar la apertura de mercados en el exterior.

Por su parte, España cuenta con la Agenda 2030 de Naciones Unidas como una de sus referencias de acción política, con especial atención al objetivo 13 de acción por el clima y reducción de emisiones, sin perder de vista los objetivos de crecimiento y de industria, innovación e infraestructuras. Así mismo, nuestro país también ha favorecido la colaboración entre empresas. Un claro ejemplo de ello se encuentra en el terreno de la aviación, donde se pretende que Indra y Airbus España colaboren conjuntamente en el desarrollo del sucesor del Eurofighter.

Para recapitular, parece claro que, para que se alcance el objetivo de que el sector industrial europeo represente el $20 \%$ del PIB en los próximos años, es necesario un mayor compromiso en la inversión de $\mathrm{I}+\mathrm{D}+\mathrm{i}$, alentando a un mayor desarrollo de Proyectos Importantes de Interés Común Europeo (PIICE), volviendo a una industria de vanguardia caracterizada por la calidad y respeto por el medio ambiente, ampliando el alcance de la circularidad de la economía e incrementando el comercio intraeuropeo. Por lo tanto, el verdadero reto para las economías es cómo reaccionar ante estos desarrollos y cambios constantes, qué enfoque dar a la política económica e industrial, identificar las medidas concretas que se deben tomar para asegurar la revitalización industrial y actuar con celeridad.

\section{Bibliografía}

ANFAC (2019). Informe Anual 2018. Recuperado de https://anfac.com/wp-content/uploads/2019/07/ ANFAC-Informe-Anual-2018.pdf

ANFAC (2019). Notas de producción y exportación. Recuperado de https://anfac.com/actualidad/ notas-de-produccion-y-exportacion/la-produccionde-vehiculos-en-espana-se-resiente-por-lacaida-de-sus-principales-mercados-deexportacion/

Comisión Europea (2016). Comunicación de la Comisión al Parlamento Europeo, al Consejo, al Comité Económico y Social Europeo y al Comité de las Regiones. Digitalización de la industria europea. Aprovechar todas las ventajas de un mercado único digital. Recuperado de https:// eur-lex.europa.eu/legal-content/ES/TXT/ PDF/?uri= CELEX:52016DC0180\&from=EN $\quad \square$ 
Comisión Europea (2019). Informe de la Comisión al Parlamento Europeo, al Consejo, al Comité Económico y Social Europeo, al Comité de las Regiones y al Banco Europeo de Inversiones sobre la ejecución del Plan de Acción Estratégico para las Baterías: creación de una cadena de valor estratégica para las baterías en Europa. Recuperado de https://eur-lex.europa.eu/resource.html?uri=cellar:72b1e42b-5ab2-11e9-915101aa75ed71a1.0017.02/DOC_1\&format=PDF

Comisión Europea (10 de septiembre de 2019). Von der Leyen: por una Unión que se esfuerza por lograr más resultados. Recuperado de https:// europa.eu/rapid/press-release_IP-19-5542_ es.htm?locale $=F R$

Comisiones Obreras, Área de Estrategias Sectoriales (2018). Situación y perspectivas en el sector del automóvil. Recuperado de https://industria. ccoo.es/9ddeee3ef0745110d18ae92f9a4bc70 6000060.pdf

Comité Europeo de las Regiones (2017). «The future of industry in Europe». Recuperado de https:// espas.secure.europarl.europa.eu/orbis/sites/ default/files/generated/document/en/report_ future_industry_form_pdf.pdf

Cruz Peña, J. (10 de octubre de 2018). Infinity Lithium busca el apoyo de Moncloa para impulsar el coche eléctrico en España. El Confidencial. Recuperado de https://www.elconfidencial.com/ empresas/2019-10-10/infinity-lihium-apoyomoncloa-impulsar-coche-electrico-espana_ 2275556/

Delgado, J., y Ferraz, E. (2014). Política de competencia, política industrial y campeones nacionales en tiempos de crisis. El derecho de la competencia en tiempos de crisis. Madrid: Fundación Rafael del Pino. Recuperado de https://www.frdelpino.es/wp-content/uploads/2014/10/es_8Juan-Delgado.pdf

EIT InnoEnergy (2018). The Strategic Action Plan on Batteries. Recuperado de https://www.innoenergy.com/about/about-innoenergy/policyresearch/the-strategic-action-plan-on-batteries/
Federal Ministry for Economic Affairs and Energy (2019). National Industrial Strategy 2030: Strategic guidelines for a German and European industrial policy. Recuperado de https://www. bmwi.de/Redaktion/EN/Publikationen/Industry/ national-industry-strategy-2030.pdf? blob=publicationFile\& $v=9$

ICEX (2018). Ficha País de Estados Unidos, indicadores económicos. Recuperado de https://www. icex.es/icex/es/navegacion-principal/todosnuestros-servicios/informacion-de-mercados/ paises/navegacion-principal/el-mercado/estudios-informes/DOC2016674009.html?idPais=US

ICEX (2018). Ficha País de Chile, indicadores económicos. Recuperado de https://www.icex.es/ icex/es/navegacion-principal/todos-nuestros-servicios/informacion-de-mercados/paises/ navegacion-principal/el-mercado/estudios-informes/DOC2016645955.html?idPais=CL\&null

ICEX (2019). Ficha País de China, indicadores económicos. Recuperado de https://www.icex.es/ icex/es/navegacion-principal/todos-nuestros-servicios/informacion-de-mercados/paises/ navegacion-principal/el-mercado/estudios-informes/DOC2016645933.html?idPais=CN

ICEX (2019). Ficha País de Corea del Sur, indicadores económicos. Recuperado de https://www. icex.es/icex/es/navegacion-principal/todosnuestros-servicios/informacion-de-mercados/ paises/navegacion-principal/el-mercado/estudios-informes/DOC2016645947.html?idPais= $\mathrm{KR}$

ICEX (2019). Ficha País de Japón, indicadores económicos. Recuperado de https://www.icex.es/ icex/es/navegacion-principal/todos-nuestros-servicios/informacion-de-mercados/paises/ navegacion-principal/el-mercado/estudios-informes/DOC2016645940.html?idPais=JP

Infinity Lithium Corporation (agosto de 2019). Pre-Feasibility Study: San Jose Valdeflorez Lithium Hydroxide Project, August. Recuperado de https://docs.wixstatic.com/ugd/b8c660_79b7117f9cb44a748aefb38fcb0b3110.pdf 
Ministerio de Asuntos Exteriores, Unión Europea y Cooperación (2019). Ficha País de Rusia, PIB por sectores de actividad y por componentes del gasto. Recuperado de http://www.exteriores. gob.es/Documents/FichasPais/RUSIA_FICHA\% 20PAIS.pdf

Ministerio de Economía, Industria y Competitividad (2017). Agenda Sectorial de la Industria de Automoción.

Ministerio de Industria, Comercio y Turismo (2019). Directrices Generales de la nueva política industrial española 2030.

Ministerio de Industria, Comercio y Turismo (2019). Vehículo Eléctrico. Economía Industrial (411).

Pizá de Silva, C. (7 de septiembre de 2019). Indra y el nuevo Eurofighter: España resucita la idea de un 'campeón nacional' en Defensa. El Confidencial. Recuperado de https://www.elconfidencial.com/ empresas/2019-09-07/defensa-tecnologiaindustria-militar-inversion_2213631/

Rifkin, J. (2011). La Tercera Revolución Industrial: cómo el poder lateral está transformando la energía, la economía y el mundo. Ediciones Paidós: Estado y Sociedad.

Scholtes Ruiz, R. (2019). Impacto del vehículo eléctrico en la industria española: disrupción económica en ciernes. Economía Industrial (411): Vehículo Eléctrico. Recuperado de https://www. mincotur.gob.es/Publicaciones/Publicacionesperiodicas/Economialndustrial/RevistaEconomialndustrial/411/SCHOLTES\%20RUIZ.pdf

Tsiropoulos, I., Tarvydas, D., y Lebedeva, N. (2018). Baterías de ion-litio para aplicaciones de movilidad $y$ de almacenamiento estacionario. Escenarios de costes y crecimiento del mercado. Recuperado de http://publications.jrc.ec.europa.eu/repository/ bitstream/JRC113360/kjna29440enn.pdf 